\title{
Möbius Transformations in Noncommutative Conformal Geometry
}

\author{
P. J. M. Bongaarts* \\ Lorentz Institute for Theoretical Physics \\ Leiden University \\ P.O. Box 9506 \\ 2300 RA Leiden, The Netherlands \\ bongaart@lorentz.leidenuniv.nl
}

\author{
J. Brodzki ${ }^{\dagger}$ \\ Department of Mathematics \\ University of Exeter \\ North Park Road \\ Exeter EX4 5HE, UK \\ brodzki@maths.ex.ac.uk
}

\begin{abstract}
We study the projective linear group $P G L_{2}(A)$ associated with an arbitrary algebra $A$, and its subgroups from the point of view of their action on the space of involutions in $A$. This action formally resembles Möbius transformations known from complex geometry. By specifying $A$ to be an algebra of bounded operators in a Hilbert space $\mathbf{H}$, we rediscover the Möbius group $\mu_{e v}(M)$ defined by Connes and study its action on the space of Fredholm modules over the algebra $A$. There is an induced action on the $K$-homology of $A$, which turns out to be trivial. Moreover, this action leads naturally to a simpler object, the polarized module underlying a given Fredholm module, and we discuss this relation in detail. Any polarized module can be lifted to a Fredholm module, and the set of different lifts forms a category, whose morphisms are given by generalized Möbius tranformations. We present an example of a polarized module canonically associated with the differentiable structure of a smooth manifold $V$. Using our lifting procedure we obtain a class of Fredholm modules characterizing the conformal structures on $V$. Fredholm modules obtained in this way are a special case of those constructed by Connes, Sullivan and Teleman.
\end{abstract}

\footnotetext{
*Supported in part by the Visitor Fund of the Department of Mathematics, University of Exeter.

†Supported in part by an LMS Scheme 4 grant and a grant from the Exeter University Research Fund.
} 


\section{Introduction}

Much of the pioneering work of Connes in noncommutative geometry rests on the notion of Fredholm module, which arises as an abstract formulation of the calculus of elliptic operators on a differentiable manifold. It has been demonstrated in numerous examples that Fredholm modules contain much of the geometric information associated with the underlying manifold. A key result in this direction is the theorem of Connes, Sullivan and Teleman $[6 \|[7]$ which associates an even Fredholm module with each oriented even dimensional conformal manifold. Conversely, given such a Fredholm module, one can reconstruct the original conformal structure [5, p.332]. It thus transpires that Fredholm modules are very close to being noncommutative analogues of conformal structures on differentiable manifolds. This fact indicates that they play a more basic role than spectral triples that capture the essence of the Riemannian geometry of smooth manifolds. As is well known, conformal structure is fundamental in general relativity, where it carries information about causality. Moreover, many interesting non-compact conformal manifolds can be conformally compactified (unlike the Riemannian case) which is a further indication that Fredholm modules will be useful in this context. They provide also a natural framework for the study of pseudo-Riemannian structures in noncommutative geometry.

The point of departure for our work is a result of Connes, which introduces a group of transformations of Fredholm modules. These transformations are formally analogous to Möbius transformations known from complex geometry. In order to provide geometric background for them we define, for any algebra $A$, the projective line $P_{1}(A)$ and the projective group $P G L_{2}(A)$. This group contains several interesting subgroups that act in various ways on the set of involutions in $A$. One of the most interesting cases in which this construction may be considered is when $A$ is an algebra of bounded operators on a Hilbert space and $M$ is its commutant. In this case it is possible to give a complete descriptions of subgroups of $P G L_{2}(A)$ that act on the space of self-adjoint involutions. Among those subgroups we discover the Möbius group $\mu_{e v}(M)$ defined by Connes. We study the structure of this group in some detail. In particular, we employ the Cayley transform to rederive the general formula for the polar decomposition of Möbius transformations. From this it follows easily that the Möbius group retracts onto the group of unitary elements in $M$. Since the same result is true for any $C^{*}$-algebra $A$, we can combine this statement with our further study of the action of the Möbius group on the space of Fredholm modules to prove that Möbius transformations act trivially on the $K$-homology of the algebra $A$.

After this basic set-up is ready, we turn to the question of the action of the Möbius group on the space of Fredholm modules. We first study in detail the structure of the space of unitarily equivalent Fredholm modules, proving a 'localization theorem'. We then discuss the action of the Möbius group. An interesting feature of the action of this group on the space of Fredholm modules 
is that it gives rise to a simpler object, the polarized module. Roughly speaking, Fredholm modules contain information about confomal structure of a space, whereas polarized modules encode its 'differential' structure. We study in detail the relation between these objects and show in particular (building on the results of [3]) that any Fredholm module has a canonical underlying polarized module. Conversely, any polarized module may be lifted to a Fredholm module. This lift is not unique, in general, and in fact we prove that all possible Fredholm module lifts of a given polarized module may be conveniently grouped together in a category, whose morphisms are generalized Möbius transformations. The most important example of a polarized module is one that can be canonically associated with the differentiable structure of a smooth manifold $V$. Any lift of this canonical polarized module to a Fredholm module depends on the choice of a conformal structure on $V$. Any Frehdolm module obtained this way turns out to be a special case of the Connes-Sullivan-Teleman Fredholm module associated with a general conformal manifold of even dimension.

Another insight gained from the study of geometric examples is that Fredholm modules are normally constructed using algebras of complex-valued functions acting in a complex Hilbert space. Thus if one is interested in real manifolds and algebras real-valued functions, it seems that part of the data is lost in the process. This suggests that there should be a property of Fredholm modules that would describe this real structure. Hence we introduce the notion of real Fredholm modules and real polarized modules and show that the action of the Möbius group preserves this notion. The canonical polarized modules and its lifts to a Fredholm module are real in this sense.

\section{The group of Möbius transformations}

\subsection{The projective line $P_{1}(A)$ and the projective group $P G L_{2}(A)$}

In this section, we set up the algebraic background for discussion of projective lines and projective groups associated with an algebra $A$. The following section will bring topological considerations, where we shall assume that the algebra $A$ is a Banach or a $C^{*}$-algebra.

Let $A$ be a complex unital algebra. We denote by $G L_{1}(A)$ the group of invertible elements in $A$. Let us introduce the following equivalence relation on the space $A \times A-\{(0,0)\}$. We say that $\left(a_{1}, b_{1}\right) \sim\left(a_{2}, b_{2}\right)$, for $a_{i}, b_{i} \in A$ iff there exists $\lambda \in G L_{1}(A)$ such that

$$
a_{1}=a_{2} \lambda, \quad b_{1}=b_{2} \lambda
$$

1.1 Definition. The quotient space $(A \times A-\{(0,0)\}) / \sim$, denoted $P_{1}(A)$, will be called the projective line over $A$. 
1.2 Remark. If the algebra $A$ is not commutative, then there are two ways to define the projective line, corresponding to the left and right actions of the group $G L_{1}(A)$ on $A \times A$.

1.3 Definition. The finite part, or set of finite points of $P_{1}(A)$, consists of the equivalence classes of pairs $(a, b)$ in which $b$ is invertible. This set will be denoted $P_{1}(A)^{f}$.

1.4 Remark. A representative $(a, b)$ of an equivalence class in $P_{1}(A)^{f}$ is clearly equivalent to the pair $\left(a b^{-1}, 1\right)$. This implies that there is a $1-1$ correspondence between elements of $P_{1}(A)^{f}$ and $A$, given by $[(a, b)] \mapsto z=a b^{-1}$.

1.5 Remark. In the case where $A$ is the field of complex numbers, the projective line is obtained from the set of 'finite' complex numbers by adding a single 'point at infinity'. In the general case one may have many elements in the algebra $A$ that are non-zero, but nevertheless not invertible, so the projective line is obtained there by adding not just a single point, but a set of 'points at infinity'.

Let $M_{2}(A)$ be the algebra of $2 \times 2$ matrices with entries in $A$, and let $G L_{2}(A)$ be the group of invertible elements of $M_{2}(A)$. Both $M_{2}(A)$ and $G L_{2}(A)$ act on the left on the space $A \times A$ by the usual matrix multiplication. Since this left action obviously commutes with the right diagonal action of the group $G L_{1}(A)$ introduced earlier, the action of $G L_{2}(A)$ descends to the projective line $P_{1}(A)$.

The induced action of $G L_{2}(A)$ on $P_{1}(A)$ is not effective, as is not difficult to see. Let $Z(A)$ be the center of the algebra $A$, i.e, $Z(A)=\{a \in A \mid a b=b a, \forall b \in$ $A\}$. It is clear that elements of $G L_{2}(A)$ of the form

$$
\left(\begin{array}{ll}
a & 0 \\
0 & a
\end{array}\right)
$$

with $a \in Z(A) \cap G L_{1}(A)$ leave any point of the projective line $P_{1}(A)$ invariant.

1.7 Lemma. Let $N$ be the subgroup of $G L_{2}(A)$ with the property that $g(x)=x$ for any $g \in N$ and $x \in P_{1}(A)$. Then

$$
N=\left\{\left(\begin{array}{cc}
a & 0 \\
0 & a
\end{array}\right) \mid a \in Z(A) \cap G L_{1}(A)\right\} .
$$

Proof. Let $T \in G L_{2}(A)$ with matrix entries $a, b, c, d$, be such that for every $\left(x_{1}, x_{2}\right) \in A \times A-\{(0,0)\}$, there exist a $\lambda \in G L_{1}(A)$ such that $T\left(x_{1}, x_{2}\right)=$ $\left(x_{1} \lambda, x_{2} \lambda\right)$. Taking for $\left(x_{1}, x_{2}\right)$ successively $(0,1),(1,0)$ and $(1,1)$ one obtains 
$b=0, c=0$ and $d=a$. Next, take a pair $(1, x)$, where $x \in A$ is arbitrary. There exists a $\lambda_{x} \in G L_{1}(A)$ such that $T(1, x)=\left(\lambda_{x}, x \lambda_{x}\right)$, which gives immediately $\lambda_{x}=a$ and then $a x=x a$. So $a x=x a$ for arbitrary $x \in A$. Finally, a diagonal matrix $T \in G L_{2}(A)$ whose diagonal entries both equal $a$ is invertible iff $a$ is invertible.

Since it is clear that $N$ is a normal subgroup of $G L_{2}(A)$, we can introduce the following definition.

1.8 Definition. The projective linear group $P G L_{2}(A)$ is by definition the quotient group

$$
P G L_{2}(A)=G L_{2}(A) / N
$$

It follows from the Lemma that the projective group $P G L_{2}(A)$ acts effectively on the projective line $P_{1}(A)$.

We shall now study various subgroups of $P G L_{2}(A)$. Our first order of business will be to identify elements from $P G L_{2}(A)$ which restrict, in some sense, to transformations of the subset $P_{1}(A)^{f}$ of finite points of the projective line.

Let then $T \in P G L_{2}(A)$ be represented by a matrix $T=\left(\begin{array}{ll}a & b \\ c & d\end{array}\right)$ with entries in $A$. Since $T$ acts on $P_{1}(A)^{f}$ by

$$
\left(\begin{array}{ll}
a & b \\
c & d
\end{array}\right)\left(\begin{array}{l}
z \\
1
\end{array}\right)=\left(\begin{array}{l}
a z+b \\
c z+d
\end{array}\right)
$$

the vector on the right is an element of $P_{1}(A)^{f}$ if and only if

$$
\left(\begin{array}{c}
a z+b \\
c z+d
\end{array}\right) \sim\left(\begin{array}{c}
(a z+b)(c z+d)^{-1} \\
1
\end{array}\right)
$$

This happens when $c z+d$ is invertible for any $z \in P_{1}(A)^{f}$. As this assumption is quite restrictive, we shall first investigate possible action of Möbius transformations on the subset of involutions in $P_{1}(A)^{f}$, i.e., elements $f$ such that $f^{2}=1$. We begin with the simplest possible case.

1.9 Lemma. Let $T \in P G L_{2}(A)$ be as above. Then the action of $T$ on the two trivial involutions in $A, f_{+}=+1$ and $f_{-}=-1$ is well defined if and only if $c+d$ and $c-d$ are invertible. Furthermore, $T f_{ \pm}=f_{ \pm}$if and only if $c=b$ and $d=a$.

Proof. The first part of the lemma follows immediately from the application of the formula

$$
z \mapsto(a z+b)(c z+d)^{-1}
$$

for the action of $T$ on $P_{1}^{f}(A)$. Note that the independence of the choice of representative in the equivalence class of $T$ is obvious. 
For the second part, applying the above formula one gets $a+b=c+d$ and $-a+b=c-d$. Addition and subtraction then gives $c=b$ and $d=a$. Independence of choice of representative is again obvious.

1.10 Remark. As projective transformations are not linear in general, the conditions for the action of $T$ on $f_{ \pm}$are independent.

Let us denote by $\mathcal{G}$ the set of matrices $\left(\begin{array}{ll}a & b \\ b & a\end{array}\right) \in G L_{2}(A)$ in which $a+b$ and $a-b$ are invertible.

1.11 Proposition. $\mathcal{G}$ is a subgroup of $G L_{2}(A)$, and its image $G$ under the canonical surjection $G L_{2}(A) \rightarrow P G L_{2}(A)$ is a subgroup of the projective linear group $P G L_{2}(A)$.

Proof. The identity matrix clearly is an element of $\mathcal{G}$, and a simple calculation shows that a product of two matrices from $\mathcal{G}$ is again a matrix in $\mathcal{G}$. An element $T \in \mathcal{G}$ is by definition an element of $G L_{2}(A)$ and therefore has an inverse $T^{-1}$ in $G L_{2}(A)$. It is clear that $T^{-1}$ is defined on the two trivial involutions $f_{ \pm}$and leaves them invariant, so $T^{-1}$ is also in $\mathcal{G}$, because of Lemma 1.9. Moreover, the kernel of the canonical homomorphism of $G L_{2}(A)$ onto $P G L_{2}(A)$ is a normal subgroup of $\mathcal{G}$. Therefore the quotient group of $\mathcal{G}$ can be identified with a subgroup $G$ of the group $P G L_{2}(A)$.

1.12 Proposition. The group $\mathcal{G}$ is isomorphic to the direct product group $G L_{1}(A) \times G L_{1}(A) \subset G L_{2}(A)$. The subgroup $G$ of the projective linear group $P G L_{2}(A)$ is isomorphic to $\left(G L_{1}(A) \times G L_{1}(A)\right) / N$.

Proof. Define $x$ and $y$ in $A$ as

$$
\begin{aligned}
& x=a+b \\
& y=a-b
\end{aligned}
$$

with the inverse relations

$$
\begin{aligned}
a & =\frac{1}{2}(x+y) \\
b & =\frac{1}{2}(x-y)
\end{aligned}
$$

The invertibility of $a+b$ and $a-b$ is equivalent to the invertibility of $x$ and $y$. Matrix multiplication expressed in these coordinates becomes $\left(x_{1}, y_{1}\right)\left(x_{2}, y_{2}\right)=$ $\left(x_{1} x_{2}, y_{1} y_{2}\right)$, where $x_{i}, y_{i} \in G L_{1}(A)$, which means that the group $\mathcal{G}$ is isomorphic to the direct product group $G L_{1}(A) \times G L_{1}(A)$. Although not necessary here, we mention that using this parametrisation it is also easy to find the inverse of a matrix in $\mathcal{G}$. Since the inverse of $(x, y)$ is $\left(x^{-1}, y^{-1}\right)$ we get that

$$
\left(\begin{array}{ll}
a & b \\
b & a
\end{array}\right)^{-1}=\frac{1}{2}\left(\begin{array}{cc}
(a+b)^{-1}+(a-b)^{-1} & (a+b)^{-1}-(a-b)^{-1} \\
(a+b)^{-1}-(a-b)^{-1} & (a+b)^{-1}+(a-b)^{-1}
\end{array}\right)
$$


To prove the statement about the subgroup $G$ of $P G L_{2}(A)$, it is sufficient to note that $N$ is a normal subgroup of $G L_{1}(A) \times G L_{1}(A)$.

We finish this discussion by recording one more simple fact.

1.14 Proposition. Let $T \in G$. Assume that $T$ is represented by a matrix $T=\left(\begin{array}{ll}a & b \\ b & a\end{array}\right)$. If, for some involution $f \in A, b f+a$ is invertible, then the image of $f$ under $T$ is an involution.

Proof. We use the formula $f \mapsto(a f+b)(b f+a)^{-1}$, which is independent of the choice of representative of $T$ in $G L_{2}(A)$. Because $f^{-1}=f$, this can be written as $f \mapsto(a f+b)\left(\left(b+a f^{-1}\right) f\right)^{-1}=(a f+b) f(a f+b)^{-1}$, which immediately gives $\left[(a f+b)(b f+a)^{-1}\right]^{2}=1$.

Let us now be a bit more daring and consider projective transformations $T$ that are defined on all involutions $f \in A$. We start with the subgroup $\mathcal{G}_{\mathcal{C}}$ of $G L_{2}(A)$ consisting of matrices $\left(\begin{array}{cc}a & b \\ b & a\end{array}\right)$ such that $b f+a$ is invertible for any involution $f$ in $A$. We shall denote by $G_{C}$ the image of $\mathcal{G}_{C}$ in $P G L_{2}(A)$.

1.15 Proposition. $\mathcal{G}_{\mathcal{C}}$ is a subgroup of $G L_{2}(A)$, and $G_{C}$ is a subgroup of $P G L_{2}(A)$ acting effectively on the set of all involutions in $A$.

Proof. Let $T_{i} \in \mathcal{G}_{\mathcal{C}}$ be given by matrices $\left(\begin{array}{cc}a_{i} & b_{i} \\ b_{i} & a_{i}\end{array}\right), i=1,2$. We need to prove that the product $T_{2} T_{1}$ is an element of $\mathcal{G}_{\mathcal{C}}$, i.e., that $b f+a$ is invertible for all involutions $f$, where

$$
a=a_{2} a_{1}+b_{2} b_{1}, \quad b=a_{2} b_{1}+b_{2} a_{1} .
$$

Let $f$ be an involution in $A$ and let $f_{1}=\left(a_{1} f+b_{1}\right)\left(b_{1} f+a_{1}\right)^{-1}$. Then it is easy to check that

$$
b f+a=\left(b_{2} f_{1}+a_{2}\right)\left(b_{1} f+a_{1}\right)
$$

hence it is invertible as the product of two invertible elements. Finally, it is clear that $N$ is a normal subgroup of $\mathcal{G}_{\mathcal{C}}$, and so the second statement follows.

To summarize, we have identified subgroups of $G L_{2}(A)$ and $P G L_{2}(A)$ that fit into the following commutative diagram

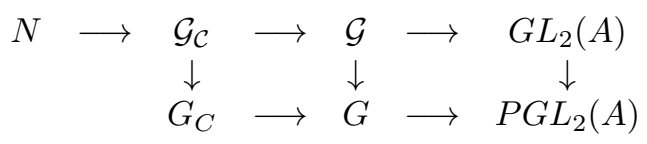


in which the horizontal arrows are inclusions and vertical arrows are surjections. The group $G$ acts effectively on the projective line $P_{1}(A)$, leaving the two trivial involutions $f_{ \pm}$fixed, whereas the subgroup $G_{C}$ acts on the set of all involutions in $A$.

Let us now assume that the algebra $A$ is $\mathbf{Z}_{2}$-graded. This grading is inherited by the matrix algebra $M_{2}(A)$, and in particular even matrices in $M_{2}(A)$ are those whose diagonal entries are even and off-diagonal entries are odd elements of $A$.

Using this additional structure we can single out a new subgroup of $\mathcal{G}$, namely $\mathcal{G}^{e v}=\mathcal{G} \cap M_{2}^{e v}(A)$. This group consists of all even matrices $\left(\begin{array}{ll}a & b \\ b & a\end{array}\right)$ in which $a+b$ and $a-b$ are invertible. These matrices preserve the trivial involutions \pm 1 . Similarly, we have the subgroup $\mathcal{G}_{C}^{e v}=\mathcal{G}_{C} \cap M_{2}^{e v}(A)$. It is straightforward to check that elements of the group $\mathcal{G}_{C}^{e v}$ map odd involutions in $A$ to odd involutions.

As before, the action of the groups $\mathcal{G}^{e v}$ and $\mathcal{G}_{C}^{e v}$ is not effective, as the elements of $N^{e v}=Z(A) \cap G L_{1}(A) \cap M_{2}^{e v}(A)$ act as identity transformations on involutions. The canonical surjection $G L_{2}(A) \rightarrow P G L_{2}(A)$ restricts to $G L_{2}(A)^{e v}$ and the kernel of this restriction is $N^{e v}$ thus giving a new group $P G L_{2}^{e v}(A)$. Note that we do not mean to imply here that $P G L_{2}(A)$ is a graded group. $N^{e v}$ is a normal subgroup of both $\mathcal{G}^{e v}$ and $\mathcal{G}_{C}^{e v}$. Thus we get two subgroups $G_{C}^{e v}$ and $G^{e v}$ of $P G L_{2}^{e v}(A)$. $G_{C}^{e v}$ acts effectively on the set of involutions, sending odd involutions to odd involutions. These new groups form a commutative diagram identical to 1.16 .

\subsection{The projective group and $*$-algebras}

From this point onwards we shall assume that $A$ is a $*$-algebra (which will become a Banach or a $C^{*}$-algebra later on), so that one can speak of selfadjoint and unitary elements of $A$. In the presence of this additional structure, our initial observations concerning the groups $\mathcal{G}, \mathcal{G}_{\mathcal{C}}, G$ and $G_{C}$ can be sharpened. In particular, we set out to investigate under what additional conditions the groups $\mathcal{G}_{\mathcal{C}}$ and $G_{C}$ will act on the set of self-adjoint involutions in $A$.

Let us begin with the subgroup $\mathcal{G}_{\mathcal{C}}$ of matrices $\left(\begin{array}{ll}a & b \\ b & a\end{array}\right)$ such that $b f+a$ is invertible for all involutions $f$ in $A$. We are looking for conditions on $a, b$ that would ensure that the involution $f^{\prime}=(a f+b)(b f+a)^{-1}$ is self-adjoint, for any self-adjoint involution $f$.

1.17 Lemma. The image $f^{\prime}=(a f+b)(b f+a)^{-1}$ of a self-adjoint involution $f$ is self-adjoint if and only if the following identities hold.

$$
\begin{aligned}
& {\left[a^{*} a-b^{*} b, f\right]=0} \\
& {\left[a^{*} b-b^{*} a, f\right]=0} \\
& {\left[a a^{*}-b b^{*}, f\right]=0} \\
& {\left[a b^{*}-b a^{*}, f\right]=0}
\end{aligned}
$$


Proof. We have already seen that $f^{\prime}$ is an involution. It will be self-adjoint iff

$$
\left((a f+b)(b f+a)^{-1}\right)^{*}=(a f+b)(b f+a)^{-1}
$$

which is equivalent to

$$
\left(f a^{*}+b^{*}\right)(b f+a)=\left(a^{*}+f b^{*}\right)(a f+b) .
$$

We rewrite the last formula as

$$
f\left(a^{*} b-b^{*} a\right) f-\left[f, a^{*} a-b^{*} b\right]-\left(a^{*} b-b^{*} a\right)=0
$$

and note that, since it is expected to hold for all self-adjoint involutions $f$ it must, in particular, hold for $-f$. Proceeding in the same way with $-f$ in place of $f$ produces a similar condition, and the two together yield

$$
\begin{aligned}
& {\left[a^{*} a-b^{*} b, f\right]=0} \\
& {\left[a^{*} b-b^{*} a, f\right]=0}
\end{aligned}
$$

To derive the latter two, we use variables $x=a+b$ and $y=a-b$ to rewrite the last two formulae in the following form

$$
\begin{aligned}
& {\left[x^{*} y+y^{*} x, f\right]=0} \\
& {\left[x^{*} y-y^{*} x, f\right]=0}
\end{aligned}
$$

which leads to a single condition $\left[x^{*} y, f\right]=0$. The inverse transformation to $f \mapsto$ $f^{\prime}$, which depends on $x^{-1}$ and $y^{-1}$, leads to a similar condition $\left[\left(x^{*}\right)^{-1} y^{-1}, f\right]=$ 0 which is equivalent to

$$
\left[x y^{*}, f\right]=0 .
$$

This in turn gives

$$
\begin{aligned}
& {\left[a a^{*}-b b^{*}, f\right]=0} \\
& {\left[a b^{*}-b a^{*}, f\right]=0 .}
\end{aligned}
$$

This finishes the proof of the lemma.

1.19 Remark. It is clear that the identities 1.18 still hold if $a$ and $b$ are replaced by $a u=u a, b u=u b$, with $u \in Z(A) \cap G L_{1}(A)$, and so they descend to the group $G_{C} \subset P G L_{2}(A)$.

In the case when $A$ is the $C^{*}$-algebra $\mathcal{L}$ of bounded operators in a Hilbert space $\mathbf{H}$ we can say a bit more. Since to any self-adjoint involution $f$ corresponds a self-adjoint projection $e=(1+f) / 2$, the formulae 1.18 imply that the same identities should hold for an arbitrary self-adjoint projection. Thus the operators on the left in 1.18 have to be multiples of the identity operator on $\mathbf{H}$. We thus have that

$$
\begin{aligned}
& a^{*} a-b^{*} b=\lambda_{1} \\
& a^{*} b-b^{*} a=i \lambda_{2} \\
& a a^{*}-b b^{*}=\lambda_{3} \\
& a b^{*}-b a^{*}=i \lambda_{4}
\end{aligned}
$$


where $\lambda_{i}$ are real numbers. Rewriting these formulae in terms of $x$ and $y$ we find

$$
\begin{aligned}
& x^{*} y=\lambda_{1}-i \lambda_{2} \\
& x y^{*}=\lambda_{3}-i \lambda_{4},
\end{aligned}
$$

or, if we use that $x$ and $y$ are invertible,

$$
\begin{aligned}
& x=\left(\lambda_{3}+i \lambda_{4}\right)\left(y^{*}\right)^{-1} \\
& y=\left(\lambda_{1}-i \lambda_{2}\right)^{-1}\left(x^{*}\right)^{-1}
\end{aligned}
$$

This gives that $\lambda_{1}=\lambda_{3}$ and $\lambda_{2}=-\lambda_{4}$ with $\lambda_{1}$ and $\lambda_{2}$ not both equal zero.

Our calculations thus demonstrate that elements of the group $\mathcal{G}_{C}$ may be written in the $x, y$ variables as $\left(x, \alpha\left(x^{*}\right)^{-1}\right)$, where $x$ is an arbitrary invertible element of $A$ and $\alpha=\lambda_{1}-i \lambda_{2}$ is an arbitrary complex number. Any such pair can be written as

$$
\left(x, \alpha\left(x^{*}\right)^{-1}\right)=(1, \alpha)\left(x,\left(x^{*}\right)^{-1}\right)=\left(x,\left(x^{*}\right)^{-1}\right)(1, \alpha) .
$$

This leads to the following statement.

1.21 Theorem. Let $A$ be the algebra of bounded operators on a Hilbert space $\mathbf{H}$. Then the group $\mathcal{G}_{\mathcal{C}}$ is the direct product $\mathcal{G}_{C}=G L_{1}(A) \times \mathbf{C}^{\times}$of the group of invertible elements in $A$ and the multiplicative group of complex numbers. The image $G_{C}$ of this group in $P G L_{2}(A)$ is the group $P G L_{1}(A)=G L_{1}(A) / \mathbf{C}^{\times}$.

Proof. The first part of the theorem has already been proved. The statement about the group $G_{C}$ follows from the fact that, if $A$ is the algebra of bounded operators on a Hilbert space, then by Schur's lemma its centre is just the field of complex numbers, so that $Z(A) \cap G L_{1}(A)=\mathbf{C}^{\times}$, in this case.

Keeping our assumption that $A$ is the algebra of bounded linear operators on a Hilbert space we note that the group $\mathcal{G}_{\mathcal{C}}$ contains an interesting subgroup, which is isomorphic to $G L_{1}(A)$ and which consists of elements $\left(x,\left(x^{*}\right)^{-1}\right), x \in$ $A$. This corresponds to the choice of $\lambda_{1}=\lambda_{3}=1$ and $\lambda_{2}=-\lambda_{4}=0$. This is precisely the group $\mu(A)$ defined by Connes [5, p. 335], which consists of matrices $\left(\begin{array}{cc}a & b \\ b & a\end{array}\right) \in G L_{2}(A)$ such that

$$
\left(\begin{array}{ll}
a & b \\
b & a
\end{array}\right)\left(\begin{array}{cc}
a^{*} & -b^{*} \\
-b^{*} & a^{*}
\end{array}\right)=\left(\begin{array}{cc}
a^{*} & -b^{*} \\
-b^{*} & a^{*}
\end{array}\right)\left(\begin{array}{ll}
a & b \\
b & a
\end{array}\right)=\left(\begin{array}{ll}
1 & 0 \\
0 & 1
\end{array}\right)
$$

We note that this condition is equivalent to Connes' identities

$$
\begin{aligned}
& a^{*} a-b^{*} b=1 \\
& a^{*} b-b^{*} a=0 \\
& a a^{*}-b b^{*}=1 \\
& a b^{*}-b a^{*}=0
\end{aligned}
$$


and which follows directly from our computations. Moreover, as these identities imply that $(a+b)^{-1}=a^{*}-b^{*}$ and $(a-b)^{-1}=a^{*}+b^{*}$, it is clear that condition 1.22 is a special case of the formula 1.13. Hence our results put Connes' construction in an interesting geometric context. The group $\mu(A)$ will be our main object of study, with the algebra $A$ to be specified.

Finally, let us assume that $A$ is a $\mathbf{Z}_{2}$-graded algebra with grading given by conjugation by a fixed involution $\gamma$. Following the procedure outlined here we uncover the group $\mu_{e v}(A)$ which is defined in the same way as $\mu(A)$ with the additional requirement that $a$ be even and $b$ be odd in $A$.

1.24 Proposition. $\mu_{\text {ev }}(A)$ is isomorphic to the subgroup of $G L_{1}(A)$ consisting of elements $x$ such that $\gamma x^{*} \gamma=x^{-1}$.

Proof. [3] Define a map $\mu_{e v}(A) \rightarrow G L_{1}(A)$ by

$$
\left(\begin{array}{cc}
a & b \\
b & a
\end{array}\right) \mapsto x=a+b
$$

with $a$ even and $b$ odd. The identities 1.23 give in this case that

$$
\gamma x^{*} \gamma=\gamma(a+b)^{*} \gamma=(a-b)^{*}=x^{-1}
$$

\subsection{The Möbius group over a $C^{*}$-algebra}

The general algebraic formalism developed in previous section becomes particularly useful when the algebra over which the Möbius group is defined is a $C^{*}$-algebra. We shall now look into possible refinements of the algebraic theory under this additional assumptions. There will be two special cases that roughly determine the scope of interesting choices of an algebra. One is the essential commutant of the representation $\pi$, and the other is the commutant of $\pi$. The first case is of interest from the point of view of $K$-homology, whereas the second is better suited to geometric applications. One of the tools that we shall use in further study of the Möbius group is the Cayley transform, and so we begin by recalling its basic properties.

1.25 Proposition. Let $\mathbf{H}$ be a (complex) Hilbert space. There is a $1-1$ correspondence between bounded invertible positive operators $Q$, and bounded selfadjoint operators $m$ with norm strictly smaller than one. This correspondence is given by the formula

$$
Q=\frac{1-m}{1+m},
$$

with the inverse relation

$$
m=\frac{1-Q}{1+Q} .
$$

The operator $Q$ is called the Cayley transform of $m$. 
It is not difficult to see that the Cayley transform of $Q^{-1}$ is $-m$. Note also that the fractional notation in the above formulae is justified, because

$$
(1-m)(1+m)=(1+m)(1-m)
$$

implies

$$
(1+m)^{-1}(1-m)=(1-m)(1+m)^{-1} .
$$

Finally, we observe that $Q$ and $m$ commute.

Let $A$ be a $C^{*}$-algebra of operators in a complex Hilbert space $\mathbf{H}$. In this case the group $\mu(A)$, defined in the previous section, will be called the Möbius group. In the graded case we shall consider the even group $\mu_{e v}(A)$. The isomorphism that identifies $\mu(A)$ with $G L_{1}(A)$, or a subgroup of $G L_{1}(A)$ in the graded case, allows one to define unitary and positive elements on $\mu(A)$, and to introduce polar decomposition of elements on the Möbius group. And so, an element $g=\left(\begin{array}{ll}a & b \\ b & a\end{array}\right)$ of the Möbius group is unitary if and only if the corresponding element $x=a+b$ is unitary in $G L_{1}(A)$. Such a $g$ is said to be positive if and only if $x$ is positive in $G L_{1}(A)$.

1.26 Proposition. An element $g \in \mu(A)$ is unitary if and only if it has the form

$$
g=\left(\begin{array}{ll}
u & 0 \\
0 & u
\end{array}\right)
$$

with $u$ a unitary operator in $A$.

Such an element $g$ is positive if and only if it has the form

$$
g=\left(\begin{array}{cc}
\left(1-m^{2}\right)^{-\frac{1}{2}} & -m\left(1-m^{2}\right)^{-\frac{1}{2}} \\
-m\left(1-m^{2}\right)^{-\frac{1}{2}} & \left(1-m^{2}\right)^{-\frac{1}{2}}
\end{array}\right)
$$

with $m$ a bounded, selfadjoint operator in A which has norm strictly smaller than one.

Proof. The first part of the proposition is clear. For the second, let us denote the image of $g$ in $G L_{1}(A)$ by $x$. We assume that $x$ is positive. We use the Cayley transform 1.25 to define a selfadjoint operator $m$, which has norm strictly smaller than 1 , by the formula

$$
m=\frac{1-x^{2}}{1+x^{2}}
$$

Continuous functional calculus on $A$ shows that $m$ is an element of $A$. Moreover, since $x$ is positive,

$$
x=\left(\frac{1-m}{1+m}\right)^{\frac{1}{2}} .
$$


which yields

$$
a=\frac{1}{2}\left(x+\left(x^{-1}\right)^{*}\right)=\left(1-m^{2}\right)^{-\frac{1}{2}}
$$

and

$$
b=\frac{1}{2}\left(x-\left(x^{-1}\right)^{*}\right)=-m\left(1-m^{2}\right)^{-\frac{1}{2}} .
$$

Conversely, if the element $g \in \mu(A)$ is determined by matrix entries $a$ and $b$ of the above form, then

$$
x=a+b=\left(1-m^{2}\right)^{-\frac{1}{2}}-m\left(1-m^{2}\right)^{-\frac{1}{2}}=\left(\frac{1-m}{1+m}\right)^{\frac{1}{2}},
$$

which shows that $x$ is positive.

There is an alternative parametrization of the positive elements of the Möbius group $\mu(A)$.

1.27 Proposition. An $g \in \mu(A)$ is positive if and only if it can be written as

$$
g=\left(\begin{array}{cc}
\cosh \omega & \sinh \omega \\
\sinh \omega & \cosh \omega
\end{array}\right)
$$

for a selfadjoint operator $\omega$ in $A$. This $\omega$ is uniquely determined by $g$.

Proof. Let a positive element $x$ of $G L_{1}(A)$ be the image of $g \in \mu(A)$. For a positive $x \in G L_{1}(A)$ there exists a unique bounded self-adjoint element $\omega$ such that $x=e^{\omega}$. The elements $g=\left(\begin{array}{ll}a & b \\ b & a\end{array}\right)$ is reconstructed from $x$ using the identities $a=\frac{1}{2}\left(x+\left(x^{*}\right)^{-1}\right)=\cosh \omega$ and $b=\frac{1}{2}\left(x-\left(x^{*}\right)^{-1}\right)=\sinh \omega$ which gives the proof of Proposition.

An arbitrary bounded invertible operator $A$ in a complex Hilbert space $\mathcal{H}$ admits two polar decompositions, i.e., it can be written either as $A=U_{L}\left(A^{*} A\right)^{\frac{1}{2}}$, or as $A=\left(A A^{*}\right)^{\frac{1}{2}} U_{R}$ with $U_{L}$ and $U_{R}$ unitary operators. Both decompositions are of course unique. Through the isomorphism between $G L_{1}(A)$ and $\mu(A)$ an arbitrary element $g$ of $\mu(A)$ has a corresponding pair of what may also be called polar decompositions.

1.28 Proposition. Every element $g$ of the Möbius group $\mu(A)$ can be written uniquely as a product of a unitary element and a positive one. In other words,

$$
g=\left(\begin{array}{ll}
a & b \\
b & a
\end{array}\right)=\left(\begin{array}{ll}
u & 0 \\
0 & u
\end{array}\right)\left(\begin{array}{cc}
\left(1-m^{2}\right)^{-\frac{1}{2}} & -m\left(1-m^{2}\right)^{-\frac{1}{2}} \\
-m\left(1-m^{2}\right)^{-\frac{1}{2}} & \left(1-m^{2}\right)^{-\frac{1}{2}}
\end{array}\right)
$$

In this $u=a\left(a^{*} a\right)^{-\frac{1}{2}}$ and $m=-a^{-1} b$. 
Similarly $g$ can be written as

$$
g=\left(\begin{array}{cc}
\left(1-m^{2}\right)^{-\frac{1}{2}} & -m\left(1-m^{2}\right)^{-\frac{1}{2}} \\
-m\left(1-m^{2}\right)^{-\frac{1}{2}} & \left(1-m^{2}\right)^{-\frac{1}{2}}
\end{array}\right)\left(\begin{array}{ll}
u & 0 \\
0 & u
\end{array}\right),
$$

where now $u=\left(a a^{*}\right)^{-\frac{1}{2}} a$ and $m=-b a^{-1}$.

Proof. The statement follows directly from Proposition 1.26 .

This result was first proved by Connes [5, Prop. 5, p. 335].

Let us denote by $\mathcal{U}$ the group of unitary elements in $G L_{1}(A)$.

1.30 Proposition. The unitary group $\mathcal{U}$ is a deformation retract of the group $\mu(A)$.

Proof. Let us denote by $g_{m}$ the matrix

$$
g_{m}=\left(\begin{array}{cc}
\left(1-m^{2}\right)^{-\frac{1}{2}} & -m\left(1-m^{2}\right)^{-\frac{1}{2}} \\
-m\left(1-m^{2}\right)^{-\frac{1}{2}} & \left(1-m^{2}\right)^{-\frac{1}{2}}
\end{array}\right)
$$

so that $g=u g_{m}$ in 1.29. Replacing $m$ by $t m, t \in[0,1]$, we obtain a continuous path of elements $g_{m, t}$ linking $g_{m}$ to the identity matrix.

1.31 REMARK. In what follows, we shall be mostly interested in the case where $A$ is identified with a $*$-subalgebra of the algebra of bounded operators on a Hilbert space by means of a faithful representation $\pi$. The Möbius group of interest in that case will be constructed in the same way as above with the assumption that the algebra $A$ is the commutant $M$ of the representation $\pi$. Our results will still apply in this case. The group $\mu(M)$ (or $\mu_{e v}(M)$ in the graded case) is the same as the group introduced by Connes in [5, p.334]. It would be natural to call this group the Connes-Möbius group, but for reasons of simplicity we shall use the name Möbius group.

\section{Möbius transformations of Fredholm modules}

\subsection{Fredholm modules}

Probably the most interesting feature of the Möbius group is the fact that it acts on the space of Fredholm modules. This action, as we shall see later, has interesting geometric consequences. But first, let us quickly review the standard notions. 
2.1 Definition. Let $A$ be an involutive algebra over C. An even Fredholm module over $A$ is given by the data $(\mathbf{H}, \pi, \gamma, F)$. Here $\mathbf{H}$ is a $\mathbf{Z}_{2}$-graded Hilbert space with the grading given by a self-adjoint involution $\gamma ; \pi$ is a faithful *representation $\pi$ of $A$ in $\mathbf{H}$ commuting with $\gamma$, and $F$ is a self-adjoint involution on $\mathbf{H}$ which anticommutes with $\gamma$. Moreover, we assume that for any $a \in A$, $[F, \pi(a)]$ is a compact operator.

An odd Fredholm module is defined in an analogous way but without the assumption that the space $\mathbf{H}$ is $\mathbf{Z}_{2}$-graded.

2.2 Remark. The presence of a self-adjoint involution $F$ anticommuting with $\gamma$ guarantees that the eigenspaces $\mathbf{H}^{0}$ and $\mathbf{H}^{1}$ are isomorphic. Conversely, any unitary operator $T: \mathbf{H}^{0} \rightarrow \mathbf{H}^{1}$ gives rise to a self-adjoint involution $F$ given by the formula

$$
F=\left(\begin{array}{cc}
0 & T^{*} \\
T & 0
\end{array}\right)
$$

Using this idea it is not difficult to show that the space of all s.a. involutions $F$ anticommuting with $\gamma$ is diffeomorphic to the group $U\left(\mathbf{H}^{0}\right)$ of unitary operators on $\mathbf{H}^{0}$.

2.3 Definition. Two Fredholm modules $\alpha_{j}=\left(\mathbf{H}_{j}, \pi_{j}, F_{j}, \gamma_{j}\right), j=1,2$, are called unitarily equivalent if and only if there exists a unitary map $U: \mathbf{H}_{1} \rightarrow \mathbf{H}_{2}$ such that

$$
F_{2}=U F_{1} U^{-1}, \quad \gamma_{2}=U \gamma_{1} U^{-1},
$$

and

$$
U \pi_{1}(a) U^{-1}=\pi_{2}(a), \forall a \in A .
$$

which will be written as $U \pi_{1} U^{-1}=\pi_{2}$.

In such a case we shall write $\alpha_{2}=U \alpha_{1} U^{-1}$.

One can also define a more general notion of isomorphism of Fredholm modules, but we shall not need it here.

It is not difficult to understand the structure of the space of unitarily equivalent Fredholm modules. Let us denote by FM the class of all odd Fredholm modules $\alpha=(\mathbf{H}, \pi, F)$. In the even case we shall use the symbol $\mathrm{FM}^{e v}$ to denote the class of all even Fredholm modules $\alpha=(\mathbf{H}, \pi, \gamma, F)$.

Two Fredholm modules $\alpha$ and $\alpha^{\prime}$ can only be unitarily equivalent if the representations $\pi$ and $\pi^{\prime}$ belong to the same unitary conjugacy class. Thus we have the following disjoint union decomposition

$$
\mathrm{FM}=\bigcup_{p} \mathrm{FM}_{p}
$$

where $p$ runs through the space of equivalence classes of representations $\pi$ and $\mathrm{FM}_{p}$ is the set of all Fredholm modules $(\mathbf{H}, \pi, F)$ in which $\pi \in p$. If we denote 
the relation of unitary equivalence of Fredholm modules by $\sim$ then we have the following simple observation:

$$
\mathrm{FM} / \sim=\bigcup_{p}\left(\mathrm{FM}_{p} / \sim\right)
$$

In the even case we have in the same way

$$
\mathrm{FM}^{e v} / \sim=\bigcup_{p}\left(\mathrm{FM}_{p}^{e v} / \sim\right)
$$

Since we only consider separable Hilbert spaces, we can describe the set of equivalence classes $\mathrm{FM}_{p} / \sim\left(\mathrm{FM}_{p}^{e v} / \sim\right.$, respectively) in a simple way. Since the reasoning is the same in the even and odd cases, we shall concentrate on the odd case. Let us fix a Hilbert space $\mathbf{H}_{p}$ equipped with a representation $\pi_{p} \in p$. We define $\mathrm{FM}_{\pi_{p}} \subset \mathrm{FM}_{p}$ to be the set of Fredholm modules $\alpha=\left(\mathbf{H}_{p}, \pi_{p}, F\right)$, where now only $F$ is variable. We remark that if $\pi, \rho \in p$ then $\mathrm{FM}_{\pi_{p}} \simeq \mathrm{FM}_{\rho_{p}}$.

Let us for the moment denote by $\sim_{p}$ the restriction of the equivalence relation $\sim$ to the set $\mathrm{FM}_{\pi_{p}}$. It follows directly from the definition that a unitary equivalence of two Fredholm modules from $\mathrm{FM}_{\pi_{p}}$ is established by means of a unitary operator $U$ from the commutant of the fixed representation $\pi_{p}$.

For each $\alpha=\left(\mathbf{H}_{\alpha}, \pi_{\alpha}, F_{\alpha}\right) \in \mathrm{FM}_{p}$ one can find a Fredholm module $\beta \in \mathrm{FM}_{\pi_{p}}$ such that $\alpha \sim \beta$. Indeed, as $\pi_{\alpha}$ and $\pi_{p}$ are unitarily equivalent representations, there exists a unitary isomorphism $U: \mathbf{H}_{\alpha} \rightarrow \mathbf{H}_{\pi_{p}}$ such that $\pi_{p}=U \pi_{\alpha} U^{-1}$. Define $\beta=\left(\mathbf{H}_{p}, \pi_{p}, U F_{\alpha} U^{-1}\right)$. It is clear that $\beta \in \mathrm{FM}_{\pi_{p}}$ and $\alpha \sim \beta$. Hence, using general properties of equivalence relations, we conclude that there exists a bijection between the sets of equivalence classes $\mathrm{FM}_{p} / \sim$ and $\mathrm{FM}_{\pi_{p}} / \sim_{p}$.

Repeating this construction in the even case we arrive at the following 'localization' result.

2.5 THEOREM. There are the following bijections of sets:

$$
\begin{gathered}
(\mathrm{FM} / \sim) \simeq \bigcup_{p}\left(\mathrm{FM}_{\pi_{p}} / \sim_{p}\right) \\
\left(\mathrm{FM}^{e v} / \sim\right) \simeq \bigcup_{p}\left(\mathrm{FM}_{\pi_{p}}^{e v} / \sim_{p}\right)
\end{gathered}
$$

where $p$ runs through the set of conjugacy classes of representations of $A$ in $\mathbf{H}$ and $\pi_{p}$ is a fixed representative of a given class $p$.

In other words, if we want to find all Fredholm modules unitarily equivalent to a given $\alpha=(\mathbf{H}, \pi, \gamma, F)$, it is sufficient to consider, without losing any information, the Fredholm modules that can be obtained by acting on $\alpha$ with unitary operators that belong to the commutant of $\pi$. 
We pass to the discussion of the action of the Möbius group on the space of Fredholm modules. This action was first introduced by Connes, and is defined as follows. If $\alpha=(\mathbf{H}, \pi, F, \gamma)$ is an even Fredholm module and $g=\left(\begin{array}{cc}a & b \\ b & a\end{array}\right)$, where $a, b \in M=(\pi(A))^{\prime}$ is an element of the Möbius group $\mu(M)$, then $g \alpha=\left(\mathbf{H}, F^{\prime}, \gamma\right)$ where $F^{\prime}=(a F+b)(b f-a)^{-1}$. As Connes shows, the Möbius group maps $p$-summable Fredholm modules to $p$-summable Fredholm modules of the same parity. There is an interesting generalization of this statement.

For any two bounded operators $P, Q$ on $\mathbf{H}$ we write $P \sim Q$ if $P$ and $Q$ differ by a compact operator.

2.6 Definition. The essential commutant of a representation $\pi$ in $\mathcal{L}$ is

$$
D_{\pi}(A)=\{x \in \mathcal{L} \mid \forall a \in A, \quad[\pi(a), x] \sim 0\}
$$

$D_{\pi}(A)$ is a $C^{*}$-subalgebra of the algebra $\mathcal{L}$ of bounded operators on $\mathbf{H}$. It is sometimes called the dual algebra of $A$, associated to the representation $\pi$. The importance of the algebra $D_{\pi}(A)$ lies in its close relation to the $K$-homology [10].

Let $A$ be a $C^{*}$-algebra, let $\pi$ be its representation in a separable Hilbert space $\mathbf{H}$, and let $(A, F, \mathbf{H})$ be a Fredholm module over $A$. We shall denote by $D=D_{\pi}(A)$ the essential commutant of $\pi(A)$ in $\mathcal{L}$. Associated with this data is the Möbius group $\mu(D)$. We have seen that $\mu(D)$ sends self-adjoint involutions to self-adjoint involutions, and so in order to understand its action on the space of Fredholm modules we need to understand its behaviour with respect to the compactness condition. First we treat the case of a Fredholm module, where we assume that the commutator $[F, x]$ is a compact operator for any element $x$ of the algebra $A$.

2.7 Proposition. Let $[F, x]$ be a compact operator for all $x \in A$. Then the same is true for $F^{\prime}=g(F)$, where $g \in \mu(D)$. In the case of an even Fredholm module, this statement holds for any $g \in \mu_{\text {ev }}(D)$.

Proof. We simply need to calculate the commutator $\left[F^{\prime}, x\right]$ in terms of $[F, x]$. We have

$$
\begin{aligned}
& {\left[(a F+b)(b F+a)^{-1}, x\right]} \\
& \quad=[a F+b, x](b F+a)^{-1}+(a F+b)\left[(b F+a)^{-1}, x\right]
\end{aligned}
$$

The first term in this sum may be written as

$$
[a F+b, x](b F+a)^{-1}=(a[F, x]+[a, x] F+[b, x])(b F+a)^{-1}
$$

whereas the second takes the form

$$
\begin{aligned}
& (a F+b)\left[(b F+a)^{-1}, x\right] \\
& \quad=-(a F+b)(b F+a)^{-1}(b[F, x]+[b, x] F+[a, x])(b F+a)^{-1}
\end{aligned}
$$


The first terms of formulae 2.8 and 2.9 combine to give

$$
\begin{array}{r}
a[F, x](b F+a)^{-1}-(a F+b)(b F+a)^{-1} b[F, x](b F+a)^{-1} \\
=\left((b F+a)^{-1}\right)^{*}[F, x](b F+a)^{-1}
\end{array}
$$

if we use that $\left(a-F^{\prime} b\right)^{-1}=(b F+a)^{*}$. The remaining terms all contain either $[a, x]$ or $[b, x]$ and so their sum is a compact operator. To summarize, we have

$$
\left[F^{\prime}, x\right]=\left((b F+a)^{-1}\right)^{*}[F, x](b F+a)^{-1}+K
$$

where $K$ is a compact operator.

2.11 Remark. Apart from the essential commutant $D_{\pi}(A)$, we can define a p-summable commutant

$$
D_{\pi}^{p}(A)=\left\{x \mid \forall a \in A, \quad[x, \pi(a)] \in \mathcal{L}^{p}(\mathbf{H})\right\}
$$

This is still a subalgebra of $\mathcal{L}$, but it need not be a $C^{*}$-subalgebra, as the Schatten ideals $\mathcal{L}^{p}$ are not norm-closed in $\mathcal{L}$. This problem disappears if we restrict our attention to the commutant $M$ of $\pi(A)$, which brings us back to the situation considered by Connes [5, p. 335]. In this case Lemma 2.7 can be improved: The group $\mu(M)$ maps $p$-summable Fredholm modules to $p$-summable Fredholm modules, for any $p \leq 1$. In the even case, the group $\mu_{e v}(M)$ acts in the same way on even Fredholm modules.

\subsection{Möbius group and $K$-homology}

The idea that gave rise to the notion of Fredholm module can be traced back to the important paper [1] of Atiyah, where he describes an abstract approach to the calculus of elliptic operators on a topological space in his search for a theory that could be considered dual to the $K$-theory. This idea was developed in papers by, among others, Baum, Connes, Douglas, Higson, Kasparov and led to a definition of $K$-homology of a $C^{*}$-algebra $A$ (cf. [2] 10]).

The starting point is the space of all Fredholm modules over a $C^{*}$-algebra $A$, represented in a fixed Hilbert space $\mathbf{H}$. On this space one defined the notion of equivalence using the already discussed unitary equivalence, triviality and homotopy of Fredolm modules. Let us introduce the latter two concepts.

An even Fredholm module $\alpha=(\mathbf{H}, \pi, F, \gamma)$ is trivial if and only if $[\pi(a), F]=$ 0 for all $a \in A$.

Two Fredholm modules $\alpha$ and $\beta$ are said to be homotopic if there exists a family $\left(\mathbf{H}, \pi, \gamma, F_{t}\right)$, where $t \in[0,1]$ and $F_{t}$ varies norm-continuously in the space of self-adjoint involutions anticommuting with $\gamma$. We keep the representation $\pi$ fixed here. These definitions are modified in the obvious way to the case of odd Fredholm modules. 
Using the notions of unitary equivalence, triviality and homotopy equivalence we define an equivalence relation on the space of Fredholm modules. The space of equivalence classes of even Fredholm modules with respect to this relation gives rise to the even $K$-homology group $K^{0}(A)$. Similarly, the same idea applied to the space of odd Fredholm modules produces the group $K^{1}(A)$. Since the Möbius group acts on Fredholm modules, it is natural to ask about the behaviour of this action from the point of view of $K$-homology.

It is clear that the Möbius group maps trivial Fredholm modules to trivial Fredholm modules. Moreover, we have the following result.

2.12 Proposition. The Möbius group $\mu(A)\left(\mu_{e v}(A)\right)$ in the even case) acts on the space of homotopy classes of Fredholm modules.

Proof. If $F_{t}$ is a norm-continuous family of involutions between $F_{0}$ and $F_{1}$, then

$$
g\left(F_{t}\right)=\left(a F_{t}+b\right)\left(b F_{t}+a\right)^{-1}
$$

is a norm-continuous path of self-adjoint involutions between $g\left(F_{0}\right)$ and $g\left(F_{1}\right)$.

2.13 TheOREM. The Möbius group $\mu(M)$ acts trivially on the K-homology of the algebra $A$.

Proof. Let $\alpha=(\mathbf{H}, \pi, F)$ be a Fredholm module and let $g \in \mu(M)$, where $M$ is the commutant of the representation $\pi(A)$. The Möbius transformation $g$ maps $\alpha$ to the Fredholm module $g(\alpha)=(\mathbf{H}, g(F), \pi)$. Assume that the polar decomposition of $g$ is $g=u g_{m}$. Then by Proposition 1.30, $g(\alpha)$ is homotopic to the Fredholm module $u \alpha u^{*}$, which belongs to the same $K$-homology class as $\alpha$. In the even case, an even Möbius transformation $g$ maps an even Fredholm module $\beta=(\mathbf{H}, \pi, F, \gamma)$ to $g(\beta)=(\mathbf{H}, \pi, g(F), g(\gamma))$, which is homotopic to the even Fredholm module

$$
u \beta u^{*}=\left(\mathbf{H}, \pi, u F u^{*}, u \gamma u^{*}\right)=\left(\mathbf{H}, \pi, u F u^{*}, \gamma\right)
$$

which belongs to the same $K$-homology class as $\beta$.

\subsection{Polarized modules}

To define a Fredholm module requires that we specify a Hilbert space $\mathbf{H}$. In many sitations, however, the Hilbert space structure is not really relevant, and what really counts is just the underlying locally convex topological space. Of course, we cannot hope that an arbitrary locally convex topological space could be used to gain useful information about geometric objects. There is, however, 
a category of spaces which are equipped with indefinite inner products, whose properties make them useful in geometric situations. These are Krein spaces.

Let us recall that a Hilbertian space $\mathcal{H}$ is a locally convex topological vector space which is isomorphic to a Hilbert space. In other words, there exists an inner product on $\mathcal{H}$ which induces the same topology.

2.14 Definition. A Krein space is a complex Hilbertian space $\mathcal{H}$, provided with a continuous hermitian form

$$
\sigma: \mathcal{H} \times \mathcal{H} \rightarrow C
$$

and such that there exists at least one compatible Hilbert space inner product $(\cdot, \cdot)$ on $\mathcal{H}$ given by

$$
(x, y)=\sigma(x, \gamma y),
$$

for all $x$ and $y$ in $\mathcal{H}$, with $\gamma$ an involution operator, selfadjoint with respect to $\sigma(\cdot, \cdot)$.

One verifies easily that the following three statements are equivalent:

1. $(\cdot, \cdot)$ is a hermitian form.

2. $\sigma(\gamma x, y)=\sigma(x, \gamma y), \forall x, y \in \mathcal{H}$.

3. $(\gamma x, y)=(x, \gamma y), \forall x, y \in \mathcal{H}$.

A compatible inner product is one that equipes the Hilbertian space $\mathcal{H}$ with a Hilbert space structure whose topology, determined by the inner product, is the same as the original locally convex topology of $\mathcal{H}$. In what follows, we shall frequently need to consider the same topological space either as a Krein space, equipped with an indefinite inner product $\sigma(\cdot, \cdot)$ or as a Hilbert space, with a Hilbert space inner product $(\cdot, \cdot)$. To distinguish between these two cases, we shall use the notation $\mathcal{H}$ for Krein spaces and $\mathbf{H}$ for Hilbert spaces.

2.15 Definition. A polarized module over a unital $*$-algebra $\mathcal{A}$ is a triple $\mathcal{P}=(\mathcal{H}, \pi, \mathcal{E})$ in which one has

1. a Krein space $(\mathcal{H}, \sigma(\cdot, \cdot))$;

2. a faithful unitarizable $*$-representation $\pi$ of $\mathcal{A}$ by continuous linear operators in $\mathcal{H}$;

3. a closed linear subspace $\mathcal{E}$ of $\mathcal{H}$, on which the hermitian form $\sigma(\cdot, \cdot)$ vanishes.

We assume, moreover, that

4. the operator $T: \mathcal{H} \rightarrow \mathcal{H}^{*}$ associated with the bilinear form $\sigma(\cdot, \cdot)$ maps the subspace $\mathcal{E}$ onto its annihilator $\mathcal{E}^{a n}$ in the topological dual $\mathcal{H}^{*}$ of the space $\mathcal{H}$; 
5. the operator $T_{a}: \mathcal{E} \rightarrow \mathcal{E}^{*}$ from $\mathcal{E}$ to its topological dual given by $\mathcal{E} \ni x \mapsto$ $\sigma(a \cdot, x)$, is compact for any $a \in \mathcal{A}$.

2.16 Remark. It would be more appropriate to use the name polarizable module, but in the interest of simplicity we shall use our current convention. This notion was first introduced in a slightly different form in [3].

We remark also that a representation is unitarizable iff there exists a Krein involution $\gamma$ such that, in the corresponding Hilbert space $\mathbf{H}_{\gamma}$, the representation $\pi$ becomes a unitary representation. A sufficient condition for this to hold is the existence of a Krein involution $\gamma$ commuting with $\pi$.

The problem of existence of unitarizable representations of $A$ is closely related to the similarity problem for a representation of a $C^{*}$-algebra, which was solved in several special cases [9] [8].

Our final remark on the definition of the polarized module is that although it may seem unnatural to single out a fixed subspace of $\mathcal{H}$, there are examples in which such a subspace appears quite naturally. For instance, in our most important case of an even dimensional smooth manifold, the Hilbertian space $\mathcal{H}$ will be the locally convex space of differential forms in the middle dimension. Then $\mathcal{E}$ is the closed subspace generated by the image of the de Rham differential.

One can introduce the notion of unitary equivalence of polarized modules which is analogous to that defined in the case of Fredholm modules. We state it here for the convenience of the reader.

2.17 Definition. Two polarized modules $\mathcal{P}_{i}=\left(\mathcal{H}_{i}, \pi_{i}, \mathcal{E}_{i}\right), i=1,2$ are unitarily equivalent iff there exists a Krein-unitary map (i.e., unitary with respect to the bilinear form $\sigma(\cdot, \cdot)) U: \mathcal{H}_{1} \rightarrow \mathcal{H}_{2}$ such that

$$
U\left(\mathcal{E}_{1}\right)=\mathcal{E}_{2}, \quad \pi_{2}=U \pi_{1} U^{-1}
$$

Polarized modules are more fundamental objects than Fredholm modules as we now set out to explain. This relation will be important when we discuss the geometric example of a compact conformal manifold. Our exposition here is a review and an extension of results from [3].

2.18 Proposition. To any even Fredholm module $\alpha=(\mathcal{H}, \gamma, \pi, F)$ one can associate a unique polarized module.

Proof. We put

$$
\sigma(x, y)=(x, \gamma y), \forall x, y \in \mathcal{H} .
$$

This is a continuous hermitian form, which is indefinite and nondegenerate. The space $\mathcal{H}$ equipped with the form $\sigma(\cdot, \cdot)$ becomes a Krein space.

Let $\mathcal{E}$ be the +1 -eigenspace of the involution $F$. Then $\mathcal{E}$ is a closed linear subspace of $\mathcal{H}$. Moreover, for $x$ and $y$ in $\mathcal{E}$ one has

$$
\sigma(x, y)=(x, \gamma y)=(F x, \gamma F y)=-(F x, F \gamma y)=-\sigma(x, y),
$$


which implies $\sigma(x, y)=0$ for all $x$ and $y$ in $\mathcal{E}$, and so the subspace $\mathcal{E}$ is totally isotropic, as required.

Since the Hilbert space $\mathbf{H}$ comes equipped with the orthogonal sum decomposition $\mathbf{H}=\mathcal{E} \oplus \gamma(\mathcal{E})$, we see that the (Hilbert space) orthogonal complement $\mathcal{E}^{\perp}$ of $\mathcal{E}$ is $\mathcal{E}^{\perp}=\gamma(\mathcal{E})$. Using this fact together with the Riesz representation theorem we conclude that the annihilator $\mathcal{E}^{a n}$ of the subspace $\mathcal{E}$ is conjugateisomorphic with $\gamma(\mathcal{E})$. In other words, for any $\xi \in \mathcal{E}^{\text {an }}$ there exists a unique $y=\gamma x \in \gamma(\mathcal{E})$, where $x \in \mathcal{E}$ such that

$$
\xi=(\cdot, y)=(\cdot, \gamma x)=\sigma(\cdot, x) .
$$

This shows that the map $T: x \mapsto \sigma(\cdot, x)$ from $\mathcal{H}$ to dual maps $\mathcal{E}$ onto $\mathcal{E}^{a n}$.

It remains to check the compactness condition. For any $a \in A$ and any $x^{\prime}, y^{\prime} \in \mathcal{E}$ we have

$$
\left(a x^{\prime}, \gamma y^{\prime}\right)=(a P x, \gamma P y)
$$

where $x, y \in \mathbf{H}$ and $P=(1+F) / 2$ is the orthogonal projection onto $\mathcal{E}$. So the compactness of the operator $T_{a}$ will be proved if we check that the operator $P \gamma a P$ is compact for any $a \in \mathcal{A}$. We have

$$
\begin{aligned}
\text { P } \gamma a P & =\gamma(1-F) a(1+F) / 4 \\
& =\gamma([F, a] F-[F, a]) / 4
\end{aligned}
$$

and so the result follows from the compactness condition satisfied by the Fredholm module.

This way we have defined a polarized module $\mathcal{P}(\alpha)=(\mathcal{H}, \pi, \mathcal{E})$ underlying the Fredholm module $\alpha$.

2.19 Proposition. Suppose that two Fredholm modules $\alpha_{1}$ and $\alpha_{2}$ are unitarily equivalent. Then the same is true of the underlying polarized modules $\mathcal{P}\left(\alpha_{1}\right)$ and $\mathcal{P}\left(\alpha_{2}\right)$. Moreover, the even unitary map $U$

$$
U: \mathcal{H}_{1} \rightarrow \mathcal{H}_{2}
$$

intertwining the representations $\pi_{1}$ and $\pi_{2}$ and the operators $F_{1}$ and $F_{2}$, becomes a Krein unitary map, mapping the subspace $\mathcal{E}_{1}$ onto the subspace $\mathcal{E}_{2}$.

Proof: Let $\alpha_{j}=\left(\mathbf{H}_{j}, \pi_{j}, F_{j}\right)$, for $j=1,2$, be two unitarily equivalent Fredholm modules. One defines again the corresponding polarized modules $\mathcal{P}_{j}=\mathcal{P}\left(\alpha_{j}\right)$, for $j=1,2$, as described above. The unitary equivalence of $\alpha_{1}$ with $\alpha_{2}$ is established by means of a map $U: \mathbf{H}_{1} \rightarrow \mathbf{H}_{2}$, which has the property $F_{2}=$ $U F_{1} U^{-1}$.

It remains to prove that $\mathcal{E}_{2}$ is the image of $\mathcal{E}_{1}$ under the map $U$. For this one observes that $x \in \mathcal{E}_{1}$ is a +1-eigenvector of $F_{1}$, i.e. $F_{1} x=x$ which is the same as $U^{-1} F_{2} U x=x$. It follows that $F_{2} U x=U x$, which means that $U x \in \mathcal{E}_{2}$. 
From this it follows that $\mathcal{E}_{2}=U\left(\mathcal{E}_{1}\right)$. The last statement about the map $U$ is clear.

It is an important fact that a polarized module can be lifted to an even Fredholm module. This lift is nonunique, in general, and the freedom of choice involved here is conveniently described by the action of the Möbius group, as we shall see shortly.

2.20 Definition. An involution operator $\gamma$ in the Krein space $\mathcal{H}$ of the polarized module $\mathcal{P}=(\mathcal{H}, \pi, \mathcal{E})$ is called compatible iff it is a Krein-self ajdoint involution commuting with the representation $\pi$, which makes $\mathcal{H}$ into a Hilbert space equipped with the inner product $(\cdot, \cdot)_{\gamma}=\sigma(\cdot, \gamma \cdot)$. The set of all compatible involutions associated with a polarized module $\mathcal{P}$ will be denoted $\Gamma_{\mathcal{P}}$.

We remark that the space of compatible involutions of a given polarized module is nonempty.

2.21 Proposition. Let $\gamma_{0}$ and $\gamma_{1}$ be two compatible involutions. Then

$$
\gamma_{1}=(1+m) \gamma_{0}(1+m)^{-1}
$$

where $m$ is a uniquely determined operator which is self-adjoint with respect to the scalar product $(\cdot, \cdot)_{0}$ and such that $\|m\|_{0}<1$. Moreover, $m$ anticommutes with $\gamma_{0}$ and belongs to the commutant $M$ of the representation $\pi$.

Proof. Let $Q=\gamma_{0} \gamma_{1}$. Then $Q$ is a bounded invertible operator, which is positive with respect to the inner product $(\cdot, \cdot)_{0}$. To check the last statement we simply note that

$$
(x, Q x)_{0}=\left(x, \gamma_{0} \gamma_{1} x\right)_{0}=\sigma\left(x, \gamma_{1} x\right)=(x, x)_{1} \geq 0
$$

We shall now use the Cayley transform, which establishes a 1-1 correspondence between bounded, positive, invertible operators $Q$ and bounded self adjoint operators $m$ of norm strictly smaller than 1. More precisely, for each operator $Q$ there exists a unique operator $m$

$$
Q=\frac{1-m}{1+m}
$$

with the inverse relation given by the formula

$$
m=\frac{1-Q}{1+Q} .
$$

Thus for a unique $m$,

$$
Q=\gamma_{0} \gamma_{1}=\frac{1-m}{1+m}
$$

and so

$$
\gamma_{1}=(1+m) \gamma_{0}(1+m)^{-1}
$$


To check that $m$ anticommutes with $\gamma_{0}$, we use that

$$
(1+Q) \gamma_{0}(1-Q)+(1-Q) \gamma_{0}(1+Q)=0
$$

which after multiplying on the left by $(1+Q)^{-1}$ gives

$$
\gamma_{0} \frac{1-Q}{1+Q}+\frac{1-Q}{1+Q} \gamma_{0}=\gamma_{0} m+m \gamma_{0}=0
$$

as required.

To show that $m$ commutes with $\pi(a)$, for all $a \in A$, we note that $Q$ has this property, and then use the Cayley transform together with the continuous functional calculus to conclude that the same is true for $m$.

2.22 Proposition. A polarized module $\mathcal{P}=(\mathcal{H}, \pi, \mathcal{E})$ can be lifted to a Fredholm module $\alpha(\mathcal{P})=(\mathbf{H}, \pi, \gamma, F)$, where $\gamma \in \Gamma_{\mathcal{P}}$.

Proof. Let us choose a compatible involution $\gamma \in \Gamma_{\mathcal{P}}$. It turns the Krein space $\mathcal{H}$ into a Hilbert space $\mathbf{H}_{\gamma}$ equipped with the inner product $(\cdot, \cdot)_{\gamma}=\sigma(\cdot, \gamma \cdot)$. The Hilbert space $\mathbf{H}_{\gamma}$ is equipped with the orthogonal direct sum decomposition $\mathbf{H}_{\gamma}=\mathcal{E} \oplus \gamma(\mathcal{E})$. Define $F$ to be +1 on $\mathcal{E}$ and -1 on $\gamma(\mathcal{E})$. It is clear that $F$ is an involution anticommuting with $\gamma$ and that it is self-adjoint with respect to the inner product $(\cdot, \cdot)_{\gamma}$.

We need to check that the commutator $[F, a]$ is a compact operator for any $a \in A$. To this end we need to use the assumption that the operator

$$
T_{a}: \mathcal{E} \rightarrow \mathcal{E}^{*} ; \quad x \mapsto \sigma(a \cdot, x)
$$

is compact for every $a \in A$. Then also the operator $x \rightarrow(a \cdot, \gamma x)_{\gamma}$ is compact, which in turns shows that the operator $P \gamma a P$, where $P=(1+F) / 2$ is the orthogonal projection onto $\mathcal{E}$, is also compact for any $a \in A$. Multiplying on the left by $\gamma$ we see that the operator

$$
\gamma P \gamma a P=(1-P) a P
$$

is compact, if we use that $\gamma P=(1-P) \gamma$. Since the above equality holds for all $a$ in $A$, taking the adjoints we see that the operator $P a(1-P)$ is also compact for all $a \in A$. Adding the two together we get that

$$
(1-P) a P+P a(1-P)=[P, a]=[F, a] / 2
$$

is compact. This gives the proof of the result.

\subsection{Lifting polarized modules}


What happens if we lift a given polarized module $\mathcal{P}=(\mathcal{H}, \mathcal{E}, \pi)$ in two different ways, by choosing two different compatible involutions $\gamma_{1}$ and $\gamma_{2}$ in $\Gamma_{\mathcal{P}}$ ?

The two choices give rise to two Fredholm modules $\alpha_{j}=\left(\mathbf{H}_{j}, \pi, \gamma_{j}, F_{j}\right), j=$ 1,2 , where $\mathbf{H}_{j}$ is the Hilbert equipped with the inner product $(\cdot, \cdot)_{j}=\sigma\left(\cdot, \gamma_{j} \cdot\right)$, and $F_{j}$ is the involution that is +1 on $\mathcal{E}$ and -1 on $\gamma(\mathcal{E})$. We are going to construct a map between these Fredholm modules in a few stages.

First we note that the two Hilbert space inner products are related in the following way. Let $x, y \in \mathcal{H}$. Then

$$
(x, y)_{1}=\sigma\left(x, \gamma_{1} y\right)=\sigma\left(x, \gamma_{2}^{2} \gamma_{1} y\right)=\left(x, \gamma_{2} \gamma_{1} y\right)_{2} .
$$

Let $Q=\gamma_{2} \gamma_{1}$. Then $Q$ is an invertible operator, which is self-adjoint with respect to the inner product $(\cdot, \cdot)_{1}$ as can be seen from the following simple calculation:

$$
(x, Q y)_{1}=\sigma\left(x, \gamma_{0} y\right)=\sigma\left(\gamma_{0} x, y\right)=\left(\gamma_{0} x, \gamma_{1} y\right)_{1}=\left(\gamma_{1} \gamma_{0} x, y\right)_{1}=(Q x, y)_{1} .
$$

Moreover, our assumptions about the form $\sigma$ imply that $Q$ is a positive operator. Let us denote by $W$ the square root of $Q$. Then, by 2.23, we may regard $W$ as a unitary operator $W: \mathbf{H}_{0} \rightarrow \mathbf{H}_{1}$.

Let $g_{21}$ be the following matrix

$$
g_{21}=\left(\begin{array}{cc}
\mathcal{A} & \mathcal{B} \\
\mathcal{B} & \mathcal{A}
\end{array}\right)=\left(\begin{array}{cc}
\frac{1}{2}(1+Q) & \frac{1}{2}(1-Q) \\
\frac{1}{2}(1-Q) & \frac{1}{2}(1+Q)
\end{array}\right)
$$

where $\mathcal{A}$ and $\mathcal{B}$ are regarded as operators from $\mathbf{H}_{1} \rightarrow \mathbf{H}_{2}$.

\subsection{Proposition.}

1. $\mathcal{A}$ and $\mathcal{B}$ commute with the representation $\pi$.

2. The operators $\mathcal{A}$ and $\mathcal{B}$ satisfy Connes identities 1.2.

3. The matrix $g_{21}$ is invertible and its inverse is given by

$$
g_{21}^{-1}=\left(\begin{array}{cc}
\mathcal{A}^{*} & -\mathcal{B}^{*} \\
-\mathcal{B}^{*} & \mathcal{A}^{*}
\end{array}\right)
$$

4. $g_{21}$ admits the following 'right' polar decomposition (with analogous formula holding for the 'left' polar decomposition)

$$
g_{21}=\left(\begin{array}{cc}
\left(1-m^{2}\right)^{-\frac{1}{2}} & m\left(1-m^{2}\right)^{-\frac{1}{2}} \\
m\left(1-m^{2}\right)^{-\frac{1}{2}} & \left(1-m^{2}\right)^{-\frac{1}{2}}
\end{array}\right)\left(\begin{array}{cc}
W & 0 \\
0 & W
\end{array}\right)
$$

where $m$ is the Cayley transform of $Q, m=(1-Q)(1+Q)^{-1}$ and $W=Q^{\frac{1}{2}}$. 
Proof. The first statement of the Proposition is obvious given that all compatible involutions commute with $\pi$. The second statement, which is checked by a simple calculation, directly implies the third. Finally, the proof of the polar decomposition 2.26 requires only a small modification of the proof of 1.29 .

We remark that the first factor on the right in formula 2.26 is just a positive Möbius transformation defined relative to the commutant of the representation $\pi$ in the Hilbert space $\mathbf{H}_{2}$, whereas the factor on the right is simply a unitary isomorphism identifying the space $\mathbf{H}_{1}$ with $\mathbf{H}_{2}$.

2.27 Definition. We shall call the matrix $g_{21}$ a generalized Möbius transformation associated with the ordered pair $\left(\gamma_{2}, \gamma_{1}\right)$ of compatible involutions from $\Gamma_{\mathcal{P}}$.

The following result is proved by an easy calculation.

2.28 Proposition. Let $\gamma_{1}, \gamma_{2}$ and $\gamma_{3}$ be compatible involutions. Then

$$
g_{31}=g_{32} g_{21} .
$$

Thus the set of all generalized Möbius transformations, parametrized by the set of compatible involutions $\Gamma_{\mathcal{P}}$, forms a groupoid. Now we construct a map between the two Fredholm modules $\alpha_{j}$, which are lifts of the given polarized module $\mathcal{P}$.

2.29 Proposition. Put

$$
g_{21}\left(F_{1}\right)=\left(\mathcal{A} F_{1}+\mathcal{B}\right)\left(\mathcal{A}+\mathcal{B} F_{1}\right)^{-1} .
$$

Then $g_{21}\left(F_{1}\right)=F_{2}$.

Proof. Let us denote by $\mathcal{S}_{ \pm}(F)$ the \pm 1 eigenspaces of the involution $F$. By definition of $F_{j}$, we have that

$$
\begin{aligned}
\mathcal{S}_{+}\left(F_{j}\right) & =\mathcal{E} \\
\mathcal{S}_{-}\left(F_{j}\right) & =\gamma_{j}(\mathcal{E})
\end{aligned}
$$

for $j=1,2$. It follows that $\mathcal{S}_{+}\left(F_{1}\right)=\mathcal{S}_{+}\left(F_{2}\right)$ and $\gamma_{2} \gamma_{1} \mathcal{S}_{-}\left(F_{1}\right)=\mathcal{S}_{-}\left(F_{2}\right)$. If we use expressions for $\mathcal{A}$ and $\mathcal{B}$, these relations can be expressed as

$$
\begin{aligned}
& (\mathcal{A}+\mathcal{B}) \mathcal{S}_{+}\left(F_{1}\right)=\mathcal{S}_{+}\left(F_{1}\right) \\
& (\mathcal{A}-\mathcal{B}) \mathcal{S}_{-}\left(F_{2}\right)=\mathcal{S}_{-}\left(F_{2}\right) .
\end{aligned}
$$

In turn, these two identities can be combined to give

$$
\left(\mathcal{A}+\mathcal{B} F_{1}\right) \mathcal{S}_{ \pm}\left(F_{1}\right)=\mathcal{S}_{ \pm}\left(F_{2}\right)
$$


which is equivalent to

$$
F_{2}=\left(\mathcal{A}+\mathcal{B} F_{1}\right) F_{1}\left(\mathcal{A}+\mathcal{B} F_{1}\right)^{-1} \text {. }
$$

This gives the formula 2.30 , if we use that $F_{1}$ is an involution.

Let $g_{21}$ be the matrix defined in 2.24 whose polar decomposition is given by 2.26. We define the following action of $g_{21}$ on the Fredholm module $\alpha_{1}$ :

$$
g_{21}\left(\alpha_{1}\right)=\left(W\left(\mathbf{H}_{1}\right), W \pi W^{-1}, W \gamma_{1} W^{-1}, g\left(F_{1}\right)\right)
$$

where $g\left(F_{1}\right)$ has the same meaning as in 2.30. We claim that $g_{21}\left(\alpha_{1}\right)=\alpha_{2}$.

We have already checked that $W: \mathbf{H}_{1} \rightarrow \mathbf{H}_{2}$ is a unitary operator and that $g_{21}\left(F_{1}\right)=F_{2}$. We need to show that $W \gamma_{1} W^{-1}=\gamma_{2}$ and that $W \pi W^{-1}=\pi$

To prove the first statement we use the Cayley transform to write $Q=$ $(1-m)(1+m)^{-1}$ which together with Proposition 2.21 gives

$$
\begin{aligned}
W \gamma_{1} W^{-1} & =\left(\frac{1-m}{1+m}\right)^{\frac{1}{2}}(1+m) \gamma_{2}(1+m)^{-1}\left(\frac{1-m}{1+m}\right)^{-\frac{1}{2}} \\
& =\left(1-m^{2}\right)^{\frac{1}{2}} \gamma_{2}\left(1-m^{2}\right)^{-\frac{1}{2}} .
\end{aligned}
$$

Since $\gamma_{2}$ anticommutes with $m$, it commutes with $m^{2}$, and by the continuous functional calculus it also commutes with $\left(1-m^{2}\right)^{\frac{1}{2}}$. This gives the required result. The second statement follows directly from Proposition 2.21 and the spectral mapping theorem.

Hence we arrive at the following result.

2.31 Proposition. Let $\alpha_{j}=\left(\mathbf{H}_{j}, \pi, \gamma_{j}, F_{j}\right), j=1,2$, be two Fredholm module lifts of a given polarized module $\mathcal{P}=(\mathcal{H}, \mathcal{E}, \pi)$. Then there exists a generalized Möbius transformation

$$
g_{21}=\left(\begin{array}{cc}
\mathcal{A} & \mathcal{B} \\
\mathcal{B} & \mathcal{A}
\end{array}\right)=\left(\begin{array}{cc}
\frac{1}{2}\left(1+\gamma_{2} \gamma_{1}\right) & \frac{1}{2}\left(1-\gamma_{2} \gamma_{1}\right) \\
\frac{1}{2}\left(1-\gamma_{2} \gamma_{1}\right) & \frac{1}{2}\left(1+\gamma_{2} \gamma_{1}\right)
\end{array}\right)
$$

such that $g_{21}\left(\alpha_{1}\right)=\alpha_{2}$.

If we now use this Proposition together with the fact that generalized Möbius transformations form a groupoid, as described in Proposition 2.28, we obtain the proof of the following description of the action of generalized Möbius transformations on the space of lifts of the polarized module $\mathcal{P}$.

2.32 Theorem. Let $\mathcal{P}=(\mathcal{H}, \mathcal{E}, \pi)$ be a polarized module. The space of all lifts $\alpha=(\mathbf{H}, \pi, \gamma, F)$ of $\mathcal{P}$ to a Fredholm module, parametrized by compatible involutions $\gamma$ from $\Gamma_{\mathcal{P}}$, forms a category in which morphisms are provided by invertible generalized Möbius transformations 2.24. 


\subsection{Real structure}

Many examples demonstrate that Fredholm modules are readily associated with $C^{*}$ algebras of complex valued functions on a manifold. However, Fredholm modules that can be associated with a real smooth manifold are equipped with additional structure which we shall call the real structure.

A real structure on an involutive algebra $A$ is a conjugate linear map $c: A \rightarrow$ $A$ with the properties $c^{2}=1 ; c(a b)=c(a) c(b)$, for all $a, b$ in $A ; c\left(a^{*}\right)=(c(a))^{*}$.

2.33 Definition. An even Fredholm module $\alpha=(\mathbf{H}, \pi, F, \gamma)$ over an algebra $A$ equipped with a real structure is called real iff it possesses a conjugation operator $C: \mathbf{H} \rightarrow \mathbf{H}$ with the following properties:

1. $C$ is conjugate linear and $C^{2}=1$;

2. $C$ is antiunitary, i.e., $(C x, C y)=(y, x)$ for all $x, y$ in $\mathbf{H}$;

3. $C \gamma=\epsilon \gamma C$, with $\epsilon$ a fixed factor equal +1 or -1 ;

4. $C \pi(a) C=\pi(c(a))$ for all $a$ in $A$.

5. $C F=F C$.

Our main example of a real Fredholm module will be associated with a conformal manifold of even degree.

In the same vein we can introduce real structure on a polarized module.

2.34 Definition. A polarized module $((\mathcal{H}, \sigma(\cdot, \cdot)), \pi, \mathcal{E})$ over an algebra with real structure $\mathcal{A}$ is called real iff it possesses a conjugation operator $C: \mathcal{H} \rightarrow \mathcal{H}$ with the following properties:

1. $C$ is conjugate-linear and has $C^{2}=1$,

2. $\sigma(C x, C y)=\epsilon \sigma(y, x)$, for all $x$ and $y$ in $\mathcal{H}$, with a fixed factor $\epsilon$ which is either +1 or -1 .

3. $C \pi(a) C=\pi(c(a))$, for all $a$ in $\mathcal{A}$,

4. $C(\mathcal{E})=\mathcal{E}$.

2.35 Proposition. Let $\alpha=(\mathcal{H}, \gamma, \pi, C)$ be a Fredholm module. Then the underlying polarized module $\mathcal{P}(\alpha)$ is also real.

Proof. The proof is a simple matter of comparing the definitions. 


\section{Conformal structures and Fredholm modules}

It has been shown by Connes, Sullivan and Teleman [6] [7] that the conformal structure on a compact oriented even dimensional manifold correspond to Fredholm modules over the algebra of smooth functions on that manifold, and in particular that such conformal structures can be reconstructed from the purely algebraic data of the Fredholm module. The formalism that has been described in the preceding section can be seen as a general approach to noncommutative conformal geometry. From this point of view, a polarized module $\mathcal{P}$ encodes certain features of a 'noncommutative differential manifold'whereas the set of Fredholm modules lying over $\mathcal{P}$ describes conformal structures on this manifold. The Möbius group, acting on the space of Fredholm modules, transforms the corresponding conformal structures in a way that cannot be achieved using orientation preserving diffeomorphisms. To appreciate the possibilities of this point of view it may be useful to see in some detail how the commutative example from ordinary differential geometry fits in this general algebraic framework.

Let $V$ be an oriented compact $2 l$-dimensional smooth manifold without boundary, and let $\mathcal{A}$ be the algebra of smooth complex-valued functions over $V$. Let us denote by $\mathcal{H}$ the space of smooth sections of the bundle $\Lambda^{l}\left(V, T_{C}^{*} V\right)$ of complexified $l$-forms on $V$. $\mathcal{H}$ is a locally convex topological space whose completion, which we shall also denote $\mathcal{H}$ is a Hilbertian space. Inside $\mathcal{H}$ there is a canonically determined subspace $\operatorname{Im} d$, which is spanned by forms of the type $d \omega$, where $d$ is the de Rham differential. Let us denote by $\mathcal{E}$ the closure of this space in $\mathcal{H}$. The space $\mathcal{H}$ is also equipped with a canonical continuous nondegenerate hermitian form

$$
\sigma(\omega, \eta)=(-i)^{l} \int_{V} \omega \wedge \bar{\eta}
$$

3.1 Lemma. The space $\mathcal{E}$ is a closed subspace, which is totally isotropic with respect to the indefinite form $\sigma(\cdot, \cdot)$.

Proof. We have

$$
\int_{V} d \omega \wedge \overline{d \eta}=\int_{V} d(\omega \wedge \overline{d \eta})=\int_{\partial V} \omega \wedge \overline{d \eta}=0 .
$$

The result now follows by the continuity of the hermitian form $\sigma(\cdot, \cdot)$.

The algebra $\mathcal{A}$ acts on the space of forms by left multiplication, as does the $C^{*}$-algebra $A$ of continous complex-valued functions on $V$. Let us denote this action by $\pi$. This data gives our most important example of a polarized module.

3.2 TheOREM. The triple $\mathcal{P}_{\text {can }}=(\mathcal{H}, \mathcal{E}, \pi)$ is a polarized module which is canonically associated with the manifold $V$. 
We stress here that to construct this polarized module we only used the differentiable structure of the manifold $V$. It is clearly an interesting question to discuss possible lifts of this module to a Fredholm module, using the lifting procedure described in the previous section. We recall that any such lift was constructed using a compatible involution $\gamma$ from the set $\Gamma_{\mathcal{P}}$, and so as a first step we should identify involutions $\gamma$ that are compatible with $\mathcal{P}_{\text {can }}$.

A Riemannian metric $g$ on $V$ is a smooth, nondegenerate symmetric tensor of order two which is positive definite everywhere. A conformal structure on $V$ is by definition an equivalence class of metrics, where $g_{1} \sim g_{2}$ iff $g_{1}=f g_{2}$ for some smooth real-valued function which is everywhere positive. A choice of a conformal structure $[g]$ on $V$ gives rise to a Hodge $*$ operator $*: \mathcal{H} \rightarrow \mathcal{H}$ which is used to define a scalar product

$$
(\omega, \eta)=\int_{V} \omega \wedge * \bar{\eta}
$$

We denote by $\mathbf{H}$ the space $\mathcal{H}$ equipped with this inner product. The Hodge operator is an involution up to a sign and so we can define the operator $\gamma=$ $(-i)^{l} *$ which is an involution. Moreover, $\gamma$ is self-adjoint with respect to both the indefinite form $\sigma(\cdot, \cdot)$ and the inner product $(\cdot, \cdot)$.

3.3 LEMMA. The involution $\gamma$ is a compatible involution associated with the polarized module $\mathcal{P}_{\text {can }}$.

Proof. This follows directly from the known properties of the Hodge $*$-operator.

The involution $\gamma$ gives the familiar orthogonal decomposition of $\mathbf{H}$ into spaces of self-dual and anti-self-dual forms:

$$
\mathbf{H}=\mathbf{H}_{0} \oplus \mathbf{H}_{1} .
$$

Let us now assume that the manifold $V$ has trivial cohomology in the middle dimension, which means that there are no harmonic $l$-forms on $V$. Then there is another decomposition of the space $\mathbf{H}$, namely

$$
\mathbf{H}=\mathcal{E} \oplus \gamma(\mathcal{E})
$$

Define $F$ to be the operator which +1 on $\mathcal{E}$ and -1 on $\gamma(\mathcal{E})$. The following result is then a direct consequence of our lifting procedure.

3.4 TheOREM. The operator $F$ is a self-adjoint involution anticommuting with $\gamma$. The data $\alpha=(\mathbf{H}, \gamma, \pi, F)$ determine a Fredholm module associated with the conformal structure $[g]$, which is a lift of the canonical polarized module $\mathcal{P}_{\text {can }}$. 
We remark that the Fredholm module constructed here is a special case of the Fredholm module defined by Connes, Sullivan and Teleman for a general even-dimensional conformal manifold [5, 6, 7].

As this theorem indicates, Fredholm modules contain information about the conformal structure of the manifold. The Möbius group, acting on the the space of Fredholm modules associated with a differentiable manifold through the various choices of conformal structure, deforms the underlying conformal structures in a way that is impossible to obtain using orientation preserving diffeomorphisms. Thus it seems plausible that Möbius transformations would have some relation to the deformation theory of conformal structures (see, e.g. [11). This point will need to be investigated further.

In the case when $V$ has nontrivial cohomology in the middle dimension, the above construction produces a pre-Fredholm module in the sense that the operator $F$ is not an involution but has the property that $1-F^{2}$ is a compact operator. (In fact it is of finite rank in this case.) As was shown by Connes in his seminal paper [4, any pre-Fredholm module can be lifted to a Fredholm module, thus yielding a Fredholm module associated with a conformal structure on $V$. Fredholm modules are clearly useful especially when one wants to construct the corresponding characters (cyclic cocycles), but it transpires that pre-Fredholm modules already contain interesting geometric information. There is a modification of the theory of Möbius transformations that fits that case, and it will be developed in a forthcoming paper.

3.5 Remark. The polarized and Fredholm modules constructed in this section are in fact real, in the sense of Definitions 2.33 and 2.34 .

3.6 REMARK. To construct a conformal structure from a given Fredholm module, Connes [5, p. 334] uses the following formula to calculate $L^{2 l}$-forms of one-forms on $V$ :

$$
\operatorname{Tr}_{\omega}\left(\left|\sum f_{i}\left[F, g_{i}\right]\right|\right)=c_{l} \int_{V}\left\|\sum f_{i} d g_{i}\right\|^{2 l}
$$

Here $\operatorname{Tr}_{\omega}$ is the Dixmier trace. We want to show that unitarily equivalent Fredholm modules give the same conformal class. But this is clear from our localization theorem 2.5, which shows that it is sufficient to prove this statement for unitary operators that belong to the commutant of a fixed representation of the algebra $\mathcal{A}$. In this case the unitary $m \in \mathcal{U}(M)$ acts by

$$
\begin{aligned}
\operatorname{Tr}_{\omega}\left(\sum f_{i}\left[F, g_{i}\right]\right) & \mapsto \operatorname{Tr}_{\omega}\left(\sum f_{i}\left[m F m^{-1}, g_{i}\right]\right) \\
& =\operatorname{Tr}_{\omega}\left(m \sum f_{i}\left[F, g_{i}\right] m^{-1}\right) \\
& =\operatorname{Tr}_{\omega}\left(\sum f_{i}\left[F, g_{i}\right]\right)
\end{aligned}
$$

Thus the norm, and therefore the associated conformal class, does not change under this transformation. 


\section{References}

[1] AтіYah, M. F., Global theory of elliptic operators, Proc. Int. Conf. On Functional Analysis and Related Topics, Univ. of Tokyo Press, Tokyo, 1970 .

[2] Baum, P. And Douglas, R., $K$-homology and index Theory; Proc. Symp. Pure Math 38 (1981), 1-57.

[3] Brodzki, J., Connes, A. And Ellwood, D., Polarized modules and Fredholm modules, Matem. fizika, analiz, geometria, 2 (1995), 15-24.

[4] Connes, A. Noncommutative differential geometry, Publ. Mathem. IHES 86 (1986)

[5] Connes, A., Noncommutative geometry, Academic Press 1994.

[6] Connes, A., Sullivan, D. and Teleman, N., Formules locales pour les classes de Pontriagin topologiques, C. R. Acad. Sci. Paris, 317 (1993), $521-526$.

[7] Connes, A., Sullivan, D. and Teleman, N. Quasiconformal mappings, operators on Hilbert space, and local formulae for characteristic classes, Topology 33 (1994), 663-681.

[8] Christensen, E. On non-self adjoint representations of operator algebras, Amer. J. Math 103 (1981) 817-833.

[9] HaAgerup, U. Solution of the similarity problem for cyclic representations of $C^{*}$-algebras, Ann. Math.118 (1983), 215-240

[10] Higson, N., $C^{*}$-algebra extension theory and duality, J. Fun. Analysis 129 (1995), 349-363.

[11] King, A. D., Kotschick, D., The deformation theory of anti-self-dual conformal structures, Math. Ann. 294 (1992), 591-609. 